\title{
Vektorisierung und Automatisierung am BEV - ein Zwischenbericht zur Erstellung von KM50-Vektor
}

\author{
Klaus Freitag ${ }^{\mathrm{a}, *}$, Gernot Felfernig ${ }^{\mathrm{a}}$, Andreas Pammer ${ }^{\mathrm{a}}$ \\ ${ }^{a}$ Bundesamt für Eich- und Vermessungswesen, Abteilung Kartographie - kartographie@bev.gv.at \\ * Corresponding author
}

Keywords: national mapping agencies, cartography, vectorization, automation

\section{Abstract:}

Die vom Bundesamt für Eich- und Vermessungswesen (BEV) herausgegebene topographische Grundkarte Österreichs im Maßstab 1:50.000 (ÖK50) basiert bislang flächendeckend auf einem Kartographischen Rasterdatenmodell (KM50R). Ein vektor-/objektbasiertes Datenmodell bietet demgegenüber mehrere Vorteile: Neben erweiterten Abfragemöglichkeiten und der Erstellung von Routennetzwerken weist dieses ein deutlich größeres Potenzial für die Etablierung automatisierter Generalisierungsprozesse auf.
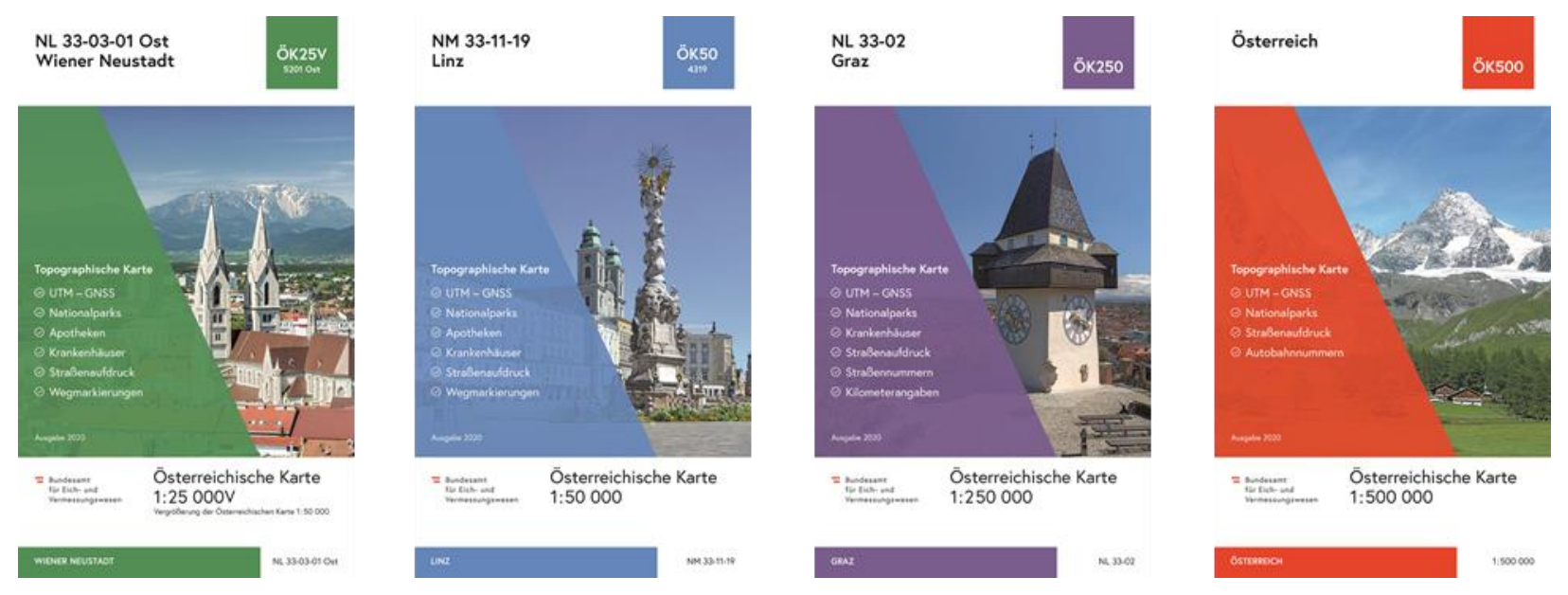

Figure 1. ÖK: Die Staatlichen Österreichischen Landkarten.

Genau dieses Ziel setzt sich das Projekt KM50-Vektor: Aus den bereits in Vektorform vorliegenden Daten des Digitalen Landschaftsmodells (DLM) soll mittels GIS-gestützter Verfahren das Kartographische Modell 1:50 000 Vektor (KM50-V) automatisiert abgeleitet werden. Dieses wird anschließend von erfahrenen Kartographen überprüft und gegebenenfalls korrigiert. Das Feedback der Kartographen fließt wiederum in die Verfeinerung der automatisierten Verfahren. Diese werden unter Berücksichtigung gängiger Geoprozessierungs-Tools in ArcGIS sowie eigens programmierter Scripts modelliert. Die große Herausforderung besteht darin, die teils subjektiv gefällten Generalisierungsentscheidungen einheitlich in maschinenlesbare Form zu bringen. Dabei müssen sowohl die Geometrie und die Topologie als auch die Sachdaten des DLM manipuliert werden.

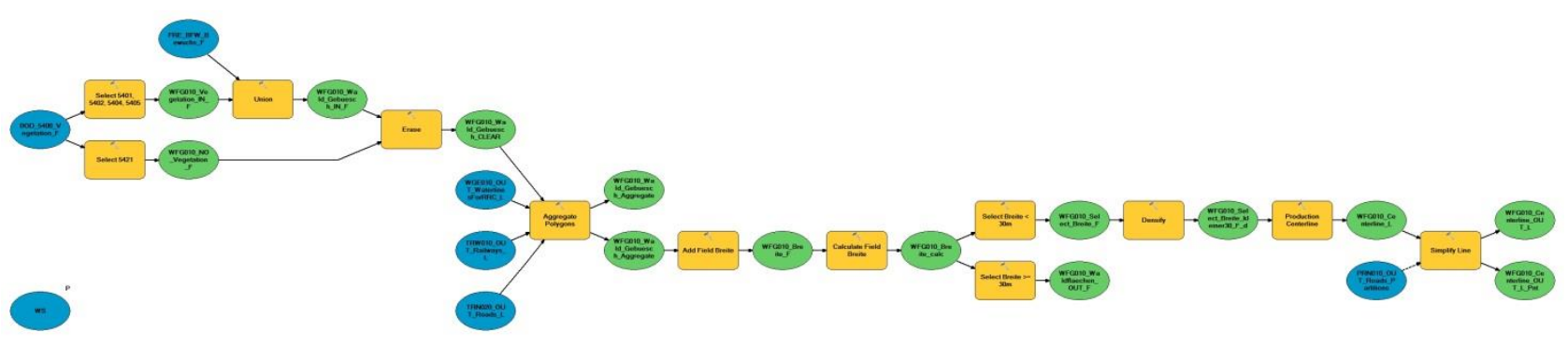

Figure 2. ArcGIS ModelBuilder. Darstellung einer Prozesskette. 
Des Weiteren entstehen bei der synchronen Bearbeitung der großen (Geo-)Datenmengen Probleme anderer Art: Häufige Abstürze aufgrund unterschiedlicher Tool-interner Topologie-Regeln oder einer Überbelastung des Arbeitsspeichers verhinderten bis vor kurzem das Rechnen größerer Ausschnitte. Durch den Einsatz zusätzlicher Werkzeuge und kartographischer Partitionen konnten die Fehler minimiert und erstmals größere Blöcke konsequent gerechnet werden. Zudem ist die Entwicklung mittlerweile soweit fortgeschritten, dass die Mehrheit der im KM50 vorkommenden Objektarten automatisiert abgeleitet werden kann.

In einem finalen Schritt werden die Modelle um die noch fehlenden Objektarten ergänzt und der Vorgang auf die gesamte Staatsfläche Österreichs ausgedehnt. Dabei ist aber zu berücksichtigen, dass einzelne wichtige Objektbereiche der Bodenbedeckung des Digitalen Landschaftsmodells (wie z.B. Einzelhäuser) zur Zeit noch flächendeckend aufgebaut werden.

Die anlaufende produktive Erstellung eines KM50-V erfolgt somit blattweise und traditionell im Aktualisierungszyklus des DLM. 2021 werden die ersten auf dem KM50-V basierenden ÖK50-Blätter gedruckt und die KM50-V-Daten über eGeodata Austria (eGA) - dem Geodaten-Portal des BEVs - verfügbar sein. Im Rahmen des Vortrags werden Voraussetzungen, Fortschritte, Zwischenergebnisse und Ausblicke des Projekts präsentiert. 